\title{
OPTICAL PROPERTIES OF NONLINEAR PERIODIC STRUCTURES
}

\author{
M.S. Malcuit and C.J. Herbert
}

Department of Physics, Lehigh University, Bethlehem, PA 18015, USA

The optical properties of nonlinear colloidal crystals in a distributed feedback configuration have been studied experimentally. Optical switching and bistability, optical limiting, and temporal instabilities have been observed in the transmission of these structures. The results of these experiments are compared to the theoretical predictions for the transmission properties of nonlinear periodic structures.

PACS numbers: $42.65 . P c, 42.70 . \mathrm{Nq}$

\section{Introduction}

There has been considerable recent interest in the study of nonlinear media whose linear index of refraction varies periodically along the direction of propagation. We will assume that the index of refraction of such a structure can be written as

$$
n(z)=n_{0}+n_{1} \cos (2 \pi z / \Lambda)+n_{2}|E|^{2},
$$

where $n_{0}$ is the bulk refractive index, $n_{1}$ is the index modulation, $n_{2}$ is the nonlinear index of refraction, and $\Lambda$ is the spatial period of the index modulation. For very low intensities when the frequency of the incident light is tuned to approximately $\omega=\pi c / n_{0} \Lambda$ the light will match the Bragg condition of the periodic structure. Under this condition light is not allowed to propagate through the periodic structure and will therefore be strongly reflected. The width of this region where light is reflected, which is referred to as the stop gap, is approximately $\Delta \omega=2 \kappa c / n_{0}$, where $\kappa=\pi n_{1} / 2 \Lambda n_{0}$ is the coupling constant of the periodic structure. The dashed line in Fig. 1 shows the low intensity transmission of a periodic structure calculated for $\kappa L=4.0$, where $L$ is the length of the structure. At high intensity the transmission of such a structure can be dramatically modified by the nonlinear contribution to the index of refraction. In this paper we describe our experimental observations of optical limiting and optical bistability with a colloidal crystal that acts as a nonlinear periodic structure $[1,2]$. In addition to these experimental results, Sankey and co-workers $[3,4]$ have observed optical switching using a corrugated silic $: n$-on-insulator waveguide as the nonlinear periodic structure. 


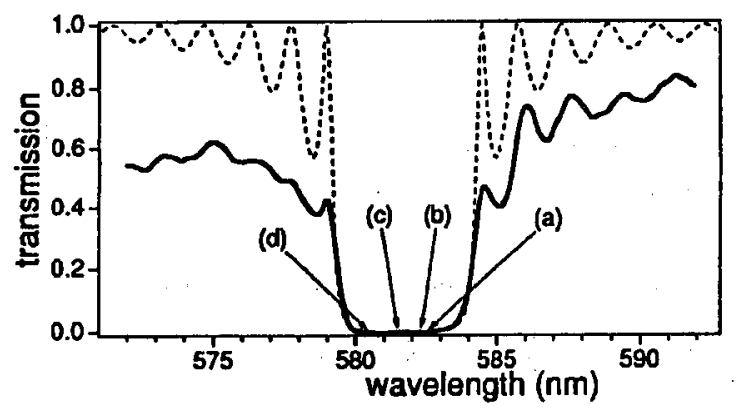

Fig. 1. The dashed curve shows the calculated transmission of a periodic structure with $\kappa L=4.0$. The solid curve shows the low intensity transmission of a colloidal crystal plotted as a function of wavelength. The arrows indicate the wavelengths of the incident light at which the transmission plots in Fig. 3 were obtained.

In steady state the transmission of a nonlinear periodic structure is predicted to exhibit optical switching, optical bistability, and optical limiting [5-9]. The characteristics of the modified transmission and its dependence on parameters such as the coupling constant of the periodic structure, the detuning of the frequency of the incident light from the center of the stop gap, and the incident intensity have been found by solving the coupled amplitude equations that describe the propagation of the forward- and backward-propagating waves in these structures [5]. If the refractive index of the periodic structure is assumed to be given by Eq. (1), and il the field of frequency $\omega$ is assumed to have both a forward- and backward-propagating component, the coupled amplitude equations can be written as

$$
\begin{aligned}
& \frac{\mathrm{d} E_{f}}{\mathrm{~d} z}=\mathrm{i} \kappa E_{b} \mathrm{e}^{-\mathrm{i} 2 \Delta \beta z}+\mathrm{i} \gamma\left(\left|E_{f}\right|^{2}+2\left|E_{b}\right|^{2}\right) E_{f}, \\
& \frac{\mathrm{d} E_{b}}{\mathrm{~d} z}=-\mathrm{i} \kappa E_{f} \mathrm{e}^{\mathrm{i} 2 \Delta \beta z}-\mathrm{i} \gamma\left(2\left|E_{f}\right|^{2}+\left|E_{b}\right|^{2}\right) E_{b},
\end{aligned}
$$

where $\Delta \beta=\beta-\beta_{0}$ is the detuning from the center of the stop gap, $\beta_{0}=\pi / \Lambda$, $\beta=n_{0} \omega / c$, and $\gamma=\pi n_{2} / \Lambda$. Figure 2 is a plot of the transmitted intensity of a nonlinear periodic structure as a function of incident intensity for the case of $\kappa L=4.0$ and a negative intensity dependent index of refraction. Figure 2 shows the behavior at three different detunings within the stop gap. For $\Delta \beta L=-4.3$ the system is predicted to exhibit simple switching as shown in Fig. 2a. When the frequency of the incident field is tuned decper into the stop gap $(\Delta \beta L=-2.0)$, the transmission is predicted to be bistable as shown in Fig. 2b. For detunings which are even deeper in the stop gap $(\Delta \beta L=2.0)$, the transmission is seen to be multistable as shown in Fig. 2c.

Along with the steady state characteristics, the temporal dependence of the transmission has been studied extensively. Under certain conditions some of the bistable states predicted by the steady state theory are found to be unstable to temporal fluctuations. Numerical calculations have shown that for a field with a frequency near the Bragg frequency and a nonlinear periodic structure with a 

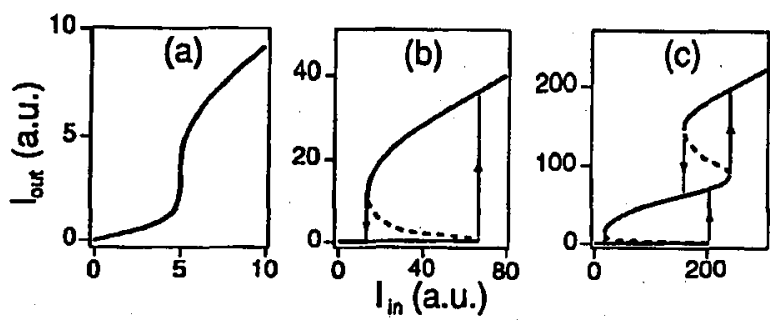

Fig. 2. The predicted transmitted intensity $\left(I_{\text {out }}\right)$ is plotted as a function of the incident intensity $\left(I_{\mathrm{m}}\right)$ for the case of $\kappa L=\mathbf{4 . 0}$ and three different detunings. (a) Simple switching for $\Delta \beta L=-4.3$, (b) bistability for $\Delta \beta L=-2.0$, (c) multistability for $\Delta \beta L=2.0$. The solid curves indicate stable states, while the dashed curves indicate unstable states. The arrows indicate where switching between transmitting states occurs.

large $\kappa$, the upper branch of the bistability curve predicted by the steady state theory is unstable and the transmission of the nonlinear periodic structure exhibits periodic self pulsing for intensities above the switching intensity [10-12]. If the incident intensity is increased to many times the switching intensity the temporal fluctuations become chaotic. A linear stability analysis performed by de Sterke [13] confirmed that there are many regions where the high-transmission state predicted by steady-state theory is temporally unstable. He found that as the frequency of the incident field is tuned deeper into the stop gap, the system tends to become more unstable.

Nonlinear periodic structures can also support soliton formation. When the frequency of the incident light is tuned outside the stop gap, soliton formation is possible due to the dispersion of the periodic structure and the nonlinearity of the media. These solitons display characteristics that are similar to those supported in nonlinear optical fibers. In this limit the nonlinear periodic structure is predicted to be useful for pulse compression [14]. When the frequency of the incident light is tuned within the stop gap, soliton formation is also possible and in this case the soliton is often referred to as a gap soliton [15-19]. A gap soliton is excited under conditions when the transmission is predicted to bistable. $A$ striking characteristic of gap solitons is that they can propagate through the nonlinear periodic structure with velocities that are much less than the group velocity of light in such medium.

\section{Experiment}

For our experimental observations of optical limiting and optical bistability we used a colloidal crystal as a nonlinear distributed feedback structure $[1,2]$. A suspension of uniform size, uniformly charged polystyrene spheres can form a periodic array. The suspensions that were used in our experiments were of either $86 \mathrm{~nm}$ or $120 \mathrm{~nm}$ diameter polystyrene spheres in water. The periodic arrays were prepared by diluting the suspensions with water. By decreasing the particle concentration the lattice spacing increases, which shifts the stop gap to longer wavelengths. The solutions were diluted such that the stop gap overlapped either 
the $514 \mathrm{~nm}$ line of an argon-ion laser or the $570-590 \mathrm{~nm}$ tuning range of a Rhodamine $6 \mathrm{G}$ dye laser. The particle concentrations used in our experiments were in the range of $7-12 \%$ by volume. For these concentrations the periodic array that is formed is a face-centered cubic structure. Once the proper concentration was achieved the suspension was placed in a $100-\mu \mathrm{m}$-thick cuvette. The lattice that is produced is oriented such that the [111] plane is oriented parallel to the faces of the cuvette. We utilize this plane for the distributed feedback.

The nonlinearity of the colloidal crystal results from the electrostrictive effect. The electrostrictive effect results in a compression of the lattice that decreases the spacing between the crystal planes. The electrostrictive effect will also increase the particle density, which will increase the average index of refraction. The decrease in the lattice spacing has the greatest affect on the modification of the transmission of the colloidal crystal. Therefore if a field of frequency $\omega$ is incident to the colloidal crystal and the intensity is increased, we expect the detuning $\Delta \beta$ from Bragg resonance to decrease as a function of intensity. Near Bragg resonance the largest electrostrictive effect is expected to result from the spatially varying electric field inside the distributed feedback structure [20]. This contribution is expected to be much larger than the electrostriction resulting from either the radial field gradient produced by the Gaussian nature of the laser beam, or the field gradient resulting from the slowly varying field amplitude along the length of the structure. For the case of two counterpropagating beams in a colloidal crystal, the ficld gradient resulting from the spatially varying ficld was calculated to be two orders of magnitude greater than the radial gradient for a spot size of approximately $10 \mu \mathrm{m}$ [21]. The theoretical study by Russell [20] showed that the field inside a periodic structure oscillates in space on the order of one grating period when the frequency of the field is near Bragg resonance. The maxima of the oscillations overlap with the high-index layer of the periodic structure when the frequency of the incident liglit is tuned to the low-frequency cdge of the stop gap. For this detuning the electrostrictive force in our system will be a minimum since the polystyrene spheres are in the high-index layer. Ilowever, as the frequency of the light is increased toward the high-frequency edge of the stop gap, the intensity maxima move away from the high-index layer until, at the high-frequency edge of the stop gap, the minima overlap the high-index layers. The electrostrictive force is therefore intensity and frequency dependent, and will increase as the detuning from the low-frequency edge of the stop gap is increased.

\section{Results and discussion}

To study optical limiting, light from an argon-ion laser was focused to a spot size of $45 \mu \mathrm{m}$ at the colloidal crystal. For the optical limiting experiment, $86 \mathrm{~nm}$ diameter spheres were used. The stop gap of the structure was tuned so that the low intensity transmission was approximately $30 \%$ at a wavelength of $514 \mathrm{~nm}$. The sample was diluted so that the wavelength of the incident light was on the high frequency side of the stop gap. Since the nonlinearity results from electrostriction we expect the stop gap to shift to higher frequencies when the incident intensity is increased. Optical limiting was observed for incident intensi- 
ties above approximately $100 \mathrm{~kW} / \mathrm{cm}^{2}$. The transmitted intensity was limited to approximately $10 \mathrm{~kW} / \mathrm{cm}^{2}$. In addition, the response time of the nonlincarity was measured to be approximately $500 \mu \mathrm{s}$.

For the optical bistability experiments, suspensions of $120 \mathrm{~nm}$ diameter particles wcre used to form the colloidal crystals. In this case the samples were diluted to a concentration such that the stop gap of the crystals overlapped the $570-590 \mathrm{~nm}$ tuning range of a Rhodamine $6 \mathrm{G}$ dye laser. The low intensity transmission of the colloidal crystal that was measured with the dye laser is shown as the solid line in Fig. 1. The coupling constant $\kappa$ of this crystal is estimated to be approximately $400 \mathrm{~cm}^{-1}$ and $\kappa L=4.0$. The switching characteristics of the colloidal crystal for various detunings within the stop gap are shown in Fig. 3 . The detunings for which

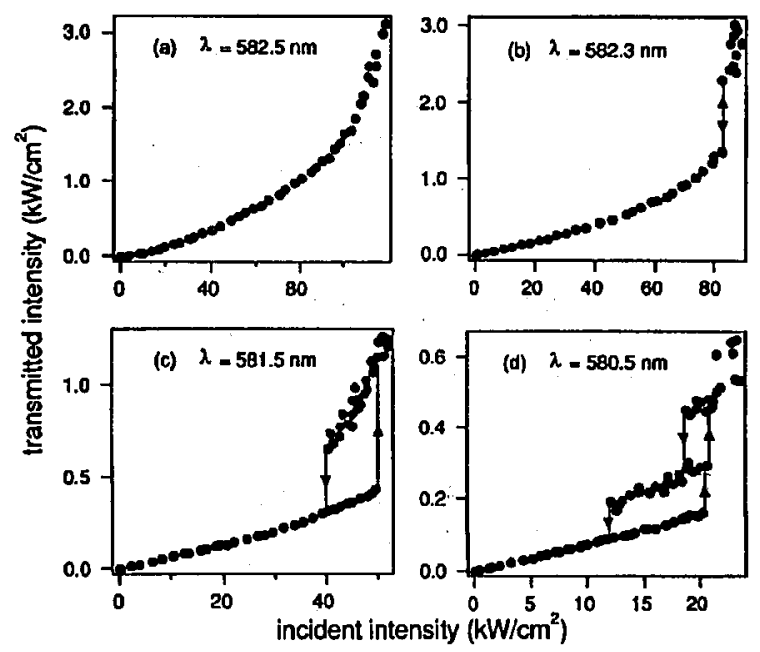

Fig. 3. Transmitted intensity plotted as a function of incident intensity for four different wavelengths. The arrows indicate where the transmission switched between different transmitting states.

the data shown in Fig. 3 were taken are marked in Fig. 1 by the letters a, b, c, and $d$. When the frequency of the incident light is tuned near the low-frequency edge of the stop gap, a nonlinear change in the transmission is obscrved as the incident intensity is increased as shown in Fig. 3a. When the frequency is tuned deeper into the stop gap the transmission shows a discontinuous increase beyond a critical incident intensity. This case is shown in Fig. 3b. For frequencies near the center of the stop gap, the transmitted intensity exhibits hysteresis and bistability as shown in Fig. 3c. If the frequency of the incident light is tuned even decper into the stop gap, additional high-transmission branches are observed. In Fig. 3d the transmission is seen to be multistable for incident intensities in the range of 18 to $20 \mathrm{~kW} / \mathrm{cm}^{2}$. The switching characteristics shown in Fig. 3 are similar to the characteristics predicted by the coupled amplitude equations for the case of a negative intensity dependent index of refraction shown in Fig. 2. As shown in Fig. 2, the 
switching intensity is expected to increase as the frequency of the incident light is tuned to higher frequencies. Ilowever, as shown in Fig. 3, we see that the switching intensity for the colloidal crystal decreases as the frequency of the incident light is increased. We believe that the detuning dependence of the electrostrictive effect discussed above, which results from the rapidly varying field inside the periodic structure, may help to explain the decrease in the switching threshold intensity.

Temporal fluctuations of the transmitted intensity were also observed when the incident intensity was increased beyond the point where bistability was observed. Figure 4 shows the transmitted intensity plotted as a function of time for an incident intensity of approximately $80 \mathrm{~kW} / \mathrm{cm}^{2}$. For Fig. 4a the wavelength of the incident light is $579.6 \mathrm{~nm}$, which is on the high-frequency side of the stop gap. As the wavelength of the incident light is tuned deeper into the stop gap the
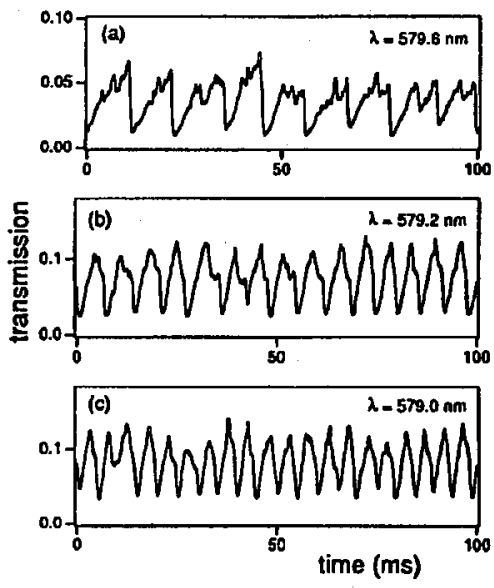

Fig. 4. Transmission of the colloidal crystal plotted as a function of time for an incident intensity of $80 \mathrm{~kW} / \mathrm{cm}^{2}$ when the wavelength of the incident laser tuned to (a) $579.6 \mathrm{~nm}$, (b) $579.2 \mathrm{~nm}$, and (c) $579.0 \mathrm{~nm}$.

frequency of the temporal fluctuations of the transmitted intensity increases as seen in Figs. $4 \mathrm{~b}$ and $4 \mathrm{c}$. In addition to the frequency of the oscillations increasing as the wavelength is tuned towards the blue, the threshold intensity at which the oscillations are observed decreases. These observations are in qualitative agreement with the linear stability analysis performed by de Sterke [13] that predicted that the oscillation frequency should increase and the threshold intensity should decrease when the frequency of the incident light is tuned deeper into the stop gap of a nonlinear periodic structure. IIe assumed that the nonlinearity resulted from an intensity dependent index of refraction with a fast response time (faster than the transit time through the sample). The response time of the nonlinearity of the colloidal crystal is approximately $500 \mu \mathrm{s}$, which is much longer than the transit time. Therefore direct comparison cannot be made between his model and the colloidal crystal system. 


\section{Conclusions}

We have observed optical limiting, and optical switching that is due to an electrostrictive nonlinearity in a colloidal crystal. Simple switching was observed near the low-frequency edge of the stop gap, while bistability occurs near the center of the stop gap. When the frequency of the incident light was tuned ncar the high-frequency edge of the stop gap multistable switching was observed. In addition, if the incident intensity is increased beyond the intensity where switching was observed, the transmitted intensity was observed to fluctuate periodically. The frequency of the oscillations increased at detunings deeper into the stop gap. These experimental observations were found to be in qualitative agreement with the thcoretical predictions for the transmission characteristics of a nonlinear periodic structure.

\section{References}

[1] C.J. Herbert, M.S. Malcuit, Opt. Lett. 17, 1037 (1992).

[2] C.J. Herbert, M.S. Malcuit, Opt. Lett. 18, 1783 (1993).

[3] N.D. Sankey, D.F. Prelewitz, T.G. Brown, Appl. Phys. Lett. 60, 1427 (1992).

[4] N.D. Sankey, D.F. Prelewitz, T.G. Brown, R.C. Tiberio, J. Appl. Phys. 73, 7111 (1993).

[5] H.G. Winful, J.H. Marburger, E. Garmire, Appl. Phys. Lett. 35, 379 (1979).

[6] H.G. Winful, Phys. Rev. Lett. 49, 1179 (1982).

[7] G. Assanto, G.I. Stcgeman, Appl. Phys. Lett. 56, 2285 (1990).

[8] P.A. Golıman, G. Bambakidis, R.J. Spry, J. Appl. Phys. 67, 40 (1989).

[9] J. He, M. Cada, IEEE J. Quantum Electron. QE-27, 1182 (1991).

[10] H.G. Winful, G.D. Cooperman, Appl. Phys. Lell. 40, 298 (1982).

[11] C.M. de Sterke, J.E. Sipc, Phys. Rev. A 42, 2858 (1990).

[12] H.G. Winful, R. Zamir, S. Feldman, Appl. Phys. Lett. 58, 1001 (1991).

[13] C.M. de Sterke, Phys. Rev. A 45, 8252 (1992).

[14] H.G. Winful, Appl. Phys. Lett. 46, 527 (1985).

[15] W. Chen, D.L. Mills, Phys. Rev. Lett. 58, 160 (1987).

[16] J.E. Sipe, H.G. Winful, Opt. Lett. 13, 132 (1988).

[17] C.M. de Sterke, J.E. Sipe, Phys. Rev. A 38, 5149 (1988).

[18] C.M. de Sterke, J.E. Sipe, Opt. Lell. 14, 871 (1989).

[19] D.N. Christodoulides, R.I. Joseph, Phys. Rev. Letl. 62, 1746 (1989).

[20] P.St.J. Russell, J. Mod. Opt. 38, 1599 (1991).

[21] C.L. Adler, N.M. Lawandy, Opt. Commun. 91, 354 (1992). 